\title{
Genetic Structure of Populations of the Pinewood Nematode Bursaphelenchus xylophilus, the Pathogen of Pine Wilt Disease, Between and Within Pine Forests
}

\author{
Zhihua Zhou, Daisuke Sakaue, Bingyun Wu, and Taizo Hogetsu
}

First author: Laboratory of Molecular Microbiology, Institute of Plant Physiology and Ecology, Shanghai Institutes for Biological Science, Chinese Academy of Science, Shanghai 200032, China; first and fourth authors: Symbiotic Function Research Unit, Asian Natural Environmental Science Center, the University of Tokyo, Midori-cho 1-1-8, Nishi-Tokyo, Tokyo 188-0002, Japan; second author: Experimental Station at Tanashi, University Forests, the University of Tokyo, Midori-cho 1-1-8, Nishi-Tokyo, Tokyo 188-0002, Japan; and third and fourth authors: Graduate School of Agricultural and Life Sciences, the University of Tokyo, Yayoi 1-1-1, Bunkyo-ku, Tokyo 1138657, Japan.

Accepted for publication 1 September 2006.

\begin{abstract}
Zhou, Z.-H., Sakaue, D., Wu, B.-Y., and Hogetsu, T. 2007. Genetic structure of populations of the pinewood nematode Bursaphelenchus xylophilus, the pathogen of pine wilt disease, between and within pine forests. Phytopathology 97:304-310.

We analyzed the genetic structure of Bursaphelenchus xylophilus populations within individual trees (subpopulations) in three distant pine forests (Tanashi, Tsukuba, and Chiba in Japan) based on the polymorphism of four microsatellite (SSR) markers. Most of the nematodes

from Tsukuba and Chiba, respectively. Despite the high genetic diversity within the Tsukuba and Chiba populations, extremely low genetic diversity was observed within the subpopulations. The genetic difference between the Tsukuba and Chiba populations was significantly smaller than that between Tanashi and either Tsukuba or Chiba. Observed heterozygosity was significantly less than expected based on Hardy-Weinberg equilibrium. These findings are best explained by a founder effect, geographic isolation between populations, explosive nematode multiplication from a small number within individual trees, and the Wahlund effect.
\end{abstract} from subpopulations in Tanashi showed the same genotype over 2 years, indicating that nematodes of that genotype dominated there for years. In contrast, 16 and 15 genotypes were identified in nematode populations
Additional keywords: Japanese pine sawyer, Monochamus alternatus, Pinus densiflora, P. thunbergii.
Pine wilt disease, caused by the pinewood nematode Bursaphelenchus xylophilus (Steiner et Buhrer) Nickle, kills large numbers of several pine species in Asia and elsewhere. Outbreaks of pine wilt disease have been a major threat to pine forests in Japan and, more recently, in China, Korea, and Portugal (27). As late as 2004 in Japan, the nematode caused an annual loss of 0.7 million $\mathrm{m}^{3}$ of pine wood (8). Although enormous efforts have been made to control pine wilt disease in the past decades, the pinewood nematode remains a serious pathogen.

The spread of pine wilt disease among trees and the development of disease within trees have been described $(2,6,15,16,27)$. The pathogenic nematodes are carried in the tracheal system of the Japanese pine sawyer beetle, Monochamus alternatus Hope, and are introduced into healthy pine trees in early summer via wounds caused by sawyer beetle maturation feeding. The introduced nematodes kill the epithelial cells in the resin canals and then invade the surrounding tissues, killing the parenchymal cells. As a result, the hydraulic conductivity of the tree is reduced. Once this happens, the nematodes reproduce and rapidly increase to large numbers within the xylem; by autumn, the tree wilts and dies. Sawyer beetles oviposit on the affected and dead trees, and their larvae develop under the bark. The next year, in early summer, nematodes move into the tracheal system of the adult beetle just before its flight from the dead tree and then are transmitted to healthy pine trees through maturation feeding by the

Corresponding author: B.-Y. Wu; E-mail address: bingyun@fr.a.u-tokyo.ac.jp

DOI: 10.1094/PHYTO-97-3-0304

(c) 2007 The American Phytopathological Society sawyer beetle. The reiteration of this cycle rapidly spreads the nematode and pine wilt disease throughout the forest.

Although the general features of $B$. xylophilus reproduction are known, many details remain unclear. Thus, we lack a detailed understanding of how the nematode infects and multiplies within trees in nature and how the sawyer beetle migrates, feeds, oviposits, and transmits nematodes in nature. Because these detailed aspects of nematode and beetle ecology are likely to determine the genetic structure of a $B$. xylophilus population within an individual host tree or a forest, we can infer the details of nematode reproduction in nature by investigating the genetic structure of natural nematode populations. Little currently is known about the genetic structure between and within nematode populations, but such information should increase our understanding of the disease.

To analyze the population genetic structure in various organisms, researchers recently have used highly polymorphic microsatellite (SSR) markers $(11,14,21,23,28,29,31-33)$. Such markers also should be useful in genetic analyses of pinewood nematode populations. Thus, in the present study, we isolated a set of polymorphic SSR markers for B. xylophilus and then used the markers to analyze the genetic structure of $B$. xylophilus populations in three pine forests.

\section{MATERIALS AND METHODS}

Collection and preparation of nematodes. B. xylophilus was collected from three pine forests suffering from pine wilt disease in the Kanto district, Japan: the Experimental Station at Tanashi, the University of Tokyo (Tanashi forest); Mount Tsukuba 
(Tsukuba forest); and the University Forest in Chiba, the University of Tokyo (Chiba forest) (Fig. 1A). The Tanashi forest is located in a western suburb of Tokyo metropolis and is surrounded by residential areas. Mountainous Tsukuba and Chiba forests are located in the northeastern part of the Kanto Plain, Ibaraki Prefecture, and in the southern part of the Boso Peninsula, Chiba Prefecture, respectively. In each forest, wood chips were collected from individual Pinus densiflora Sieb. et Zucc. (Japanese red pine) and P. thunbergii Parl. (Japanese black pine) trees killed by natural infection of the pinewood nematode. Chips were collected by drilling portions ( $18 \mathrm{~mm}$ in diameter) from the trunk (three portions per trunk) at $\approx 1.3 \mathrm{~m}$ height; the three portions were combined to form one sample per tree with $\approx 60 \mathrm{~cm}^{3}$ of wood chips per sample. In Tanashi, we sampled six $P$. densiflora and three $P$. thunbergii trees in 2001, and four $P$. densiflora and one $P$. thunbergii tree in 2002 . Twelve trees sampled from Tanashi were within an area of $\approx 200$ by $300 \mathrm{~m}$ in the Experimental Station, and the other two $P$. densiflora trees were in a temple garden $\approx 0.7 \mathrm{~km}$ from the Experimental Station. In 2001, we sampled $10 P$. densiflora trees in Tsukuba (Fig. 1B) and seven $P$. densiflora and two P. thunbergii trees in Chiba (Fig. 1C).

Nematodes were extracted from $\approx 30 \mathrm{~cm}^{3}$ of wood chips of each pine tree using a Baermann funnel (20). Nematodes extracted from a single tree were regarded as a subpopulation, and subpopulations from Tanashi, Tsukuba, and Chiba were labeled with a prefix (TA, TS, and $\mathrm{CH}$, respectively) followed by a number. All subpopulations in a forest were collectively considered to represent the forest population. Extracted nematodes were used immediately for DNA extraction without any multiplication step.

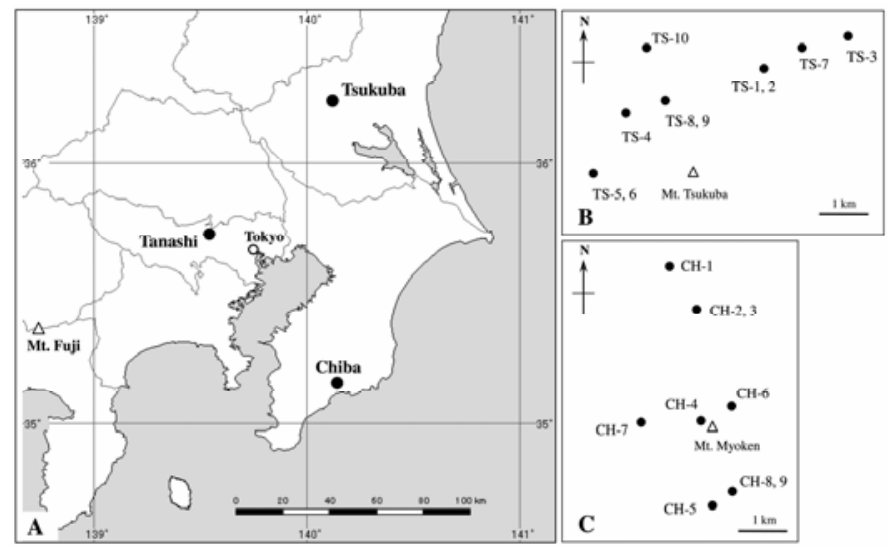

Fig. 1. A, Location of the studied forests, and sampling sites of Bursaphelenchus xylophilus subpopulations in B, Tsukuba and $\mathbf{C}$, Chiba. The nematodes extracted from an individual tree were regarded as a subpopulation, and subpopulations from Tsukuba and Chiba were labeled with a prefix (TS and $\mathrm{CH}$, respectively) followed by a number.
To test the SSR markers (see below), we used nine B. xylophilus isolates obtained from many different areas in Japan (Table 1). Seven of the nine isolates (isolates $67, \mathrm{C} 14-5, \mathrm{Ka}-4, \mathrm{OKD}-1$, S6-1, S-10, and T4) were provided by the Forestry and Forest Products Research Institute, Japan. Two of the nine B. xylophilus isolates (TES-2 and TES-4) were obtained from dead pine trees in Tanashi in 2000. Five other nematode species (including B. mисronatus, Aphelenchoides spp., Aphelenchus spp., and a species of Rhabditida) also were used to test the SSR markers. An isolate of B. mucronatus was provided by the Forestry and Forest Products Research Institute, Japan. Isolates of an Aphelenchoides sp. (Aphelenchoides sp. 1) and an Aphelenchus sp. were obtained from dead pine trees in 1997. In 2001, nematodes of another Aphelenchoides sp. (Aphelenchoides sp. 2) and a Rhabditida species were extracted from a dead pine tree in Tanashi and in Chiba, respectively, and directly used for DNA extraction. Other than these latter two nematode species, the nematodes (nine isolates of B. xylophilus and one isolate each of B. mucronatus, Aphelenchoides sp. 1, and Aphelenchus sp.) were maintained and multiplied on the fungus Botrytis cinerea growing on potato dextrose agar (PDA; Difco Laboratories, Detroit, MI). Nematodes were collected from the PDA using a Baermann funnel (20) before DNA extraction.

DNA extraction. Isolate T4 of Bursaphelenchus xylophilus was used for mass DNA extraction to develop SSR markers. Nematodes multiplied on Botrytis cinerea cultures were suspended in $200 \mu \mathrm{l}$ of water, frozen with liquid nitrogen, and ground into powder using a mortar and pestle. The powder then was suspended in $500 \mu \mathrm{l}$ of lysis buffer $(10 \mathrm{mM}$ Tris- $\mathrm{HCl}, 100 \mathrm{mM}$ EDTA, $20 \mathrm{ng}$ of RNAse A/ml, and $0.5 \%$ sodium dodecyl sulfate) and incubated at $37^{\circ} \mathrm{C}$ for $1 \mathrm{~h}$. After addition of proteinase $\mathrm{K}$ to a final concentration of $100 \mu \mathrm{g} / \mathrm{ml}$, the mixture was incubated at $50^{\circ} \mathrm{C}$ for over $3 \mathrm{~h}$. After twice partitioning with a chloroform/ isoamyl alcohol mixture (24:1, vol/vol), DNA in the upper phase was precipitated by adding three volumes of $99.5 \%$ ethanol and $1 / 10$ volume of $3 \mathrm{M} \mathrm{NaOAc}(\mathrm{pH} 5.2)$. Precipitated DNA was dissolved in Tris-EDTA (TE) buffer and stored at $-30^{\circ} \mathrm{C}$ until use.

For SSR polymorphism analyses, DNA was extracted from individual nematodes that were directly extracted from wood chips. A nematode was picked up using a fine needle (a modified dental root canal needle; Pierce, Tokyo) under a dissection microscope and transferred to a drop of TE buffer $(6 \mu \mathrm{l})$ on a glass slide. The nematode was smashed by covering it with a coverslip ( 8 by $8 \mathrm{~mm}$ ) and tapping lightly on the slip with a metal probe under the dissection microscope. Tissue of the smashed nematode was scraped into the TE buffer and collected in a microtube. This tissue suspension was used directly as the DNA template for polymerase chain reaction (PCR).

PCR amplification. SSR markers were amplified by PCR in $10 \mu \mathrm{l}$ of a reaction solution containing $1 \mu \mathrm{l}$ of template DNA solution, $0.4 \mathrm{mM}$ each dNTP, $1 \times$ buffer (Takara Shuzo Co., Shiga,

TABLE 1. Microsatellite (SSR) genotypes of nine Bursaphelenchus xylophilus isolates

\begin{tabular}{|c|c|c|c|c|c|c|c|c|c|}
\hline \multirow[b]{2}{*}{ Isolate } & \multirow[b]{2}{*}{ Collection site ${ }^{b}$} & \multicolumn{8}{|c|}{ Sizes of PCR products at each locus ${ }^{\mathrm{a}}$} \\
\hline & & $\mathrm{Bx} 01$ & $\mathrm{Bx} 02$ & $\mathrm{Bx} 03$ & Вx04 & $\mathrm{Bx} 05$ & $\mathrm{Bx} 06$ & $\mathrm{Bx} 07$ & Bx08 \\
\hline 67 & Ashikita Town, Kumamoto Pref. & $140 / 142$ & 72 & $196 / 320$ & 95 & $149 / 153$ & 139 & $150 / 154$ & $109 / 110$ \\
\hline $\mathrm{C} 14-5$ & Ichinomiya Town, Chiba Pref. & 142 & - & 228 & 99 & 153 & 135 & 154 & 109 \\
\hline $\mathrm{Ka}-4$ & Kasama City, Ibaraki Pref. & 140 & 84 & 214 & 135 & 149 & 141 & 156 & 114 \\
\hline OKD-1 & Okayama City, Okayama Pref. & 142 & 108 & 200 & 103 & 153 & 137 & 152 & 109 \\
\hline S6-1 & Mito City, Ibaraki Pref. & 146 & 76 & 214 & 135 & 149 & 141 & 155 & 109 \\
\hline S-10 & Hirose Town, Shimane Pref. & 146 & 76 & 214 & 135 & 149 & 141 & 155 & 109 \\
\hline $\mathrm{T} 4$ & Ichinoseki City, Iwate Pref. & 140 & 84 & 214 & 131 & 149 & 139 & 157 & 114 \\
\hline TES-2 & Nishitokyo City, Tokyo Metropolis & 146 & 84 & 320 & 135 & 149 & 139 & 155 & 114 \\
\hline TES-4 & Nishitokyo City, Tokyo Metropolis & 146 & 84 & 320 & 135 & 149 & 139 & 155 & 114 \\
\hline
\end{tabular}

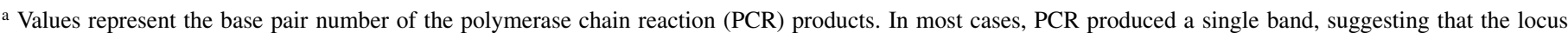
was homozygous, and only one value is listed. In a few cases, PCR produced two bands, indicating that the locus was heterozygous, and two values separated by a slash are listed; - indicates that no band was produced.

b Collection sites were widely distributed over those areas in Japan infested with B. xylophilus; Pref. = Prefecture.
} 
Japan), which included $2.5 \mathrm{mM} \mathrm{Mg}^{2+}$ and $0.5 \mathrm{U}$ of LA Taq DNA polymerase (Takara Shuzo Co.), and $0.4 \mu \mathrm{M}$ of a primer pair in which the reverse primer was labeled with Texas Red. PCR was performed in a thermal cycler (TP3000; Takara Shuzo Co.) using 29 cycles of $1 \mathrm{~min}$ at $94^{\circ} \mathrm{C}, 30 \mathrm{~s}$ at annealing temperature (Ta), and $1 \mathrm{~min}$ at $72^{\circ} \mathrm{C}$, followed by one cycle of $1 \mathrm{~min}$ at $94^{\circ} \mathrm{C}, 30 \mathrm{~s}$ at $\mathrm{Ta}$, and $5 \mathrm{~min}$ at $72^{\circ} \mathrm{C}$.

Development of SSR markers. SSR markers were isolated using a dual suppression PCR method (14). Five restrictionfragment libraries were constructed by digesting DNA from isolate T4 using the restriction enzymes AfaI, AluI, EcoRV, HaeIII, and SspI (Takara Shuzo Co.). The digested DNA fragments then were ligated with an uneven-length adaptor (26). In the first step, the library of DNA fragments digested by EcoRV was used as a template for PCR using an SSR motif primer, $(\mathrm{CA})_{10}$ or $(\mathrm{GA})_{10}$, and an adaptor primer. Amplified fragments were subcloned, and several clones were sequenced. Two specific primers for nested PCR in the second step were designed from the region flanking the SSR motif in each sequence. In the second step, nested PCR was performed on every restriction-fragment library with the two primers designed in the first step and two adaptor primers designed for nested PCR. Nested PCR products of a restriction-fragment library that showed a single clear band on agarose gel were subcloned and sequenced. An SSR marker, (i.e., a primer pair for amplifying the region containing an SSR) was designed from the sequence (Table 2).

Polymorphism analysis. Each SSR marker was amplified by PCR of nematode DNA using the SSR primer pair. A portion of the PCR product was denatured at $94^{\circ} \mathrm{C}$ for $5 \mathrm{~min}$ and separated on a sequencing gel made of $6 \%$ Long Ranger acrylamide (FMC BioProducts, Rockland, ME), 6.1 M urea, and 1.2× TBE $(0.1 \mathrm{M}$ Tris [hydroxymethyl] aminomethane, $3.0 \mathrm{mM}$ EDTA, and $0.1 \mathrm{M}$ boric acid) using a sequencer (SQ-5500; Hitachi, Tokyo). Banding pattern polymorphisms were analyzed using FRAGLYS 3.0 software (Hitachi Electronics Engineering, Tokyo). When two bands were detected, each band was considered to represent an allele at the locus; when one band was detected, it was considered to represent a homozygous allele pair. Samples with identical banding patterns at every locus were assumed to belong to the same genotype.

Statistics. The observed heterozygosity $(\mathrm{Ho})$ at each SSR locus was obtained by adding $H o$ values of all subpopulations and dividing by 33 , the number of subpopulations. The expected heterozygosity $(\mathrm{He})$ was calculated from allele frequencies at each SSR locus by Nei's equation (19). For calculating $\mathrm{He}$, each allele frequency was calculated by adding frequencies of the allele in all subpopulations and dividing by the number of subpopulations. Deviation of $\mathrm{Ho}$ from $\mathrm{He}$ (i.e., from Hardy-Weinberg equilibrium) was tested statistically by a $\chi^{2}$ test at $P<0.05$ (19).
Nei's average number of differences and mean number of pairwise differences were calculated from genotypes of individual nematodes by ARLEQUIN version 2.0 (25) as genetic differences between and within subpopulations or populations.

A dendrogram was produced with a neighbor-joining method based on Nei's average number of differences between subpopulations using Molecular Evolutionary Genetics Analysis (MEGA) version 2.1 software (13).

Population structure based on genotypic data also was inferred with STRUCTURE version 2 (22). In this program, which uses a model-based clustering method, the number of original populations $(K)$ is arbitrarily presumed. For each value of $K$, STRUCTURE then calculates the probability of the actual genotypic frequencies. The $K$ value with the highest probability and clearest genetic structure is considered to best represent reality. The program can assign individuals to the original populations and can identify migrants or admixed individuals. In this study, the program was run with the admixture model and was used without prior population information. Each value of $K(2$ to 5$)$ was run for 50,000 iterations (with a burn-in period of 50,000 iterations).

\section{RESULTS}

Isolation and characterization of SSR markers for the pinewood nematode. In the first step, 12 fragments with GT or CT repeats on one side were sequenced; in the second step, specific primers for nested PCR were designed for 9 of the 12 fragments. Eight SSR markers were successfully developed and designated as $\mathrm{Bx} 01, \mathrm{Bx} 02, \mathrm{Bx} 03, \mathrm{Bx} 04, \mathrm{Bx} 05, \mathrm{Bx} 06, \mathrm{Bx} 07$, and Bx08 (Table 2).

PCR amplifications of all eight SSR markers yielded positive electrophoresis bands from the DNA of all nine B. xylophilus isolates. DNA templates from all isolates except isolate 67 produced single electrophoresis bands for every marker, indicating that every marker was homozygous in all isolates (Table 1). For all nine isolates of $B$. xylophilus, three alleles were identified for $\mathrm{Bx} 01$, four for $\mathrm{Bx} 02$, five for $\mathrm{Bx} 03$, five for $\mathrm{Bx} 04$, two for $\mathrm{Bx} 05$, four for $\mathrm{Bx} 06$, six for $\mathrm{Bx} 07$, and three for $\mathrm{Bx} 08$ (Table 1). The nine isolates were separated into seven different genotypes by the eight markers; S6-1 and S-10, and TES-2 and TES-4 showed the same genotypes. Because the quantity of DNA from a single nematode often was insufficient for analysis by more than four markers, and because the seven genotypes could be distinguished by four markers $(\mathrm{Bx} 02, \mathrm{Bx} 03, \mathrm{Bx} 04$, and $\mathrm{Bx} 07)$, these four markers were used for subsequent polymorphism analyses.

To determine the specificity of the SSR markers to B. xylophilus, PCR amplification of six loci (Bx02, Bx03, Bx04, Bx06, $\mathrm{Bx} 07$, and $\mathrm{Bx} 08$ ) was performed for templates from five nematode species other than B. xylophilus: B. mucronatus, two

TABLE 2. Microsatellite (SSR) markers for Bursaphelenchus xylophilus ${ }^{\mathrm{a}}$

\begin{tabular}{|c|c|c|c|}
\hline Locus name & Sequences of primer pair $\left(5^{\prime}-3^{\prime}\right)$ & Repeat & Annealing temperature $\left({ }^{\circ} \mathrm{C}\right)$ \\
\hline$\overline{\mathrm{Bx} 01}$ & F-CTGACAATCTTCTGTCGTATG & & \\
\hline $\mathrm{Bx} 02$ & F-TCTGATTCTCTCACACCCTC & & \\
\hline \multirow[t]{2}{*}{$\mathrm{Bx} 03$} & F-GCCTCAAGACATTCCGAATACC & & \\
\hline & R-TCTGGTACCACGCACGTTTTC & $(\mathrm{TC})_{40}$ & 58 \\
\hline \multirow[t]{2}{*}{$\mathrm{Bx} 04$} & F-TTTCTTCTGGCATGGCAAAG & & \\
\hline & R-AAGATATTGAACGATCTTTGTGGC & $(\mathrm{TC})_{24}$ & 55 \\
\hline \multirow[t]{2}{*}{ Bx06 } & F-CGAAGAAGATCTTTAATCATCCG & & \\
\hline & R-TGAGAAATTGCAAAGAATCCTCCG & $(\mathrm{GT})_{8}$ & 55 \\
\hline \multirow[t]{2}{*}{$\mathrm{Bx} 07$} & F-AACGGAAAAGAGTCCTCACG & & \\
\hline & R-TAGGCCCTCCTTGACAAAAGC & $(\mathrm{TC})_{10}$ & 56 \\
\hline \multirow[t]{2}{*}{$\mathrm{Bx} 08$} & F-CTGCCTATTTTCGACTTCTC & & \\
\hline & R-CAAGGATCGTGTTCCTCTTTTTG & $(\mathrm{CT})_{10}$ & 55 \\
\hline
\end{tabular}

a These SSR markers were developed from isolate T4 of B. xylophilus; ... indicates that there was an intervening sequence. 
Aphelenchoides spp., an Aphelenchus sp., and a species of Rhabditida. Most of these species occur with B. xylophilus in nature (12). PCR amplification of templates from these nematodes did not yield any products. Based on these limited data, the six markers seemed to be specific to B. xylophilus.

In total, six alleles for $\mathrm{Bx} 02$, nine for $\mathrm{Bx} 03$, four for $\mathrm{Bx} 04$, and three for $\mathrm{Bx} 07$ were detected from the three forests. No more than three alleles were identified at each locus in any subpopulation (Table 3). Ho significantly deviated from $\mathrm{He}$ at every locus (Table 4).

Genetic structure of nematode subpopulations in Tanashi. In all, 420 individual nematodes (30 from every subpopulation) from Tanashi were analyzed using the four SSR markers; 418 individuals showed the same genotype, with complete homozy-

TABLE 3. Microsatellite (SSR) genotypes of Bursaphelenchus xylophilus in subpopulations ${ }^{\mathrm{a}}$

\begin{tabular}{|c|c|c|c|c|c|c|c|}
\hline \multirow[b]{2}{*}{ Site, tree number ${ }^{c}$} & \multirow[b]{2}{*}{ Sampling year } & \multirow[b]{2}{*}{ Tree species } & \multirow[b]{2}{*}{ Number of nematodes } & \multicolumn{4}{|c|}{ Sizes of PCR products in each locus ${ }^{b}$} \\
\hline & & & & $\mathrm{Bx} 02$ & $\mathrm{Bx} 03$ & $\mathrm{Bx} 04$ & $\mathrm{Bx} 07$ \\
\hline \multicolumn{8}{|l|}{ Tanashi } \\
\hline TA-1 & 2001 & Pinus densiflora & 30 & 84 & 320 & 135 & 155 \\
\hline TA-2 & 2001 & P. densiflora & 30 & 84 & 320 & 135 & 155 \\
\hline TA-3 & 2001 & P. densiflora & 30 & 84 & 320 & 135 & 155 \\
\hline TA-4 & 2001 & P. densiflora & 30 & 84 & 320 & 135 & 155 \\
\hline TA-5 & 2001 & P. densiflora & 30 & 84 & 320 & 135 & 155 \\
\hline TA-6 & 2001 & P. densiflora & 30 & 84 & 320 & 135 & 155 \\
\hline TA-7 & 2001 & P. thunbergii & 30 & 84 & 320 & 135 & 155 \\
\hline TA-8 & 2001 & P. thunbergii & 30 & 84 & 320 & 135 & 155 \\
\hline TA-9 & 2001 & P. thunbergii & 30 & 84 & 320 & 135 & 155 \\
\hline TA-10 & 2002 & P. densiflora & 30 & 84 & 320 & 135 & 155 \\
\hline TA-11 & 2002 & P. thunbergii & 30 & 84 & 320 & 135 & 155 \\
\hline \multirow[t]{3}{*}{ TA-12 } & 2002 & P. densiflora & 1 & $76 / 84$ & 320 & 135 & 155 \\
\hline & & & 1 & 84 & 322 & 135 & 155 \\
\hline & & & 28 & 84 & 320 & 135 & 155 \\
\hline TA-13 & 2002 & P. densiflora & 30 & 84 & 320 & 135 & 155 \\
\hline TA-14 & 2002 & P. densiflora & 30 & 84 & 320 & 135 & 155 \\
\hline \multicolumn{8}{|l|}{ Tsukuba } \\
\hline TS-1 & 2001 & P. densiflora & 26 & 84 & 214 & 117 & 155 \\
\hline \multirow[t]{3}{*}{ TS-2 } & 2001 & P. densiflora & 2 & $72 / 76$ & 214 & 135 & 147 \\
\hline & & & 8 & 84 & 214 & 135 & 147 \\
\hline & & & 1 & 84 & 214 & 135 & $147 / 155$ \\
\hline TS-3 & 2001 & P. densiflora & 48 & 84 & 214 & 135 & 155 \\
\hline TS-4 & 2001 & P. densiflora & 26 & 86 & 214 & 135 & 155 \\
\hline \multirow[t]{3}{*}{ TS-5 } & 2001 & P. densiflora & 4 & 84 & 214 & 135 & 155 \\
\hline & & & 24 & 84 & $214 / 320$ & 135 & 155 \\
\hline & & & 2 & 84 & 320 & 135 & 155 \\
\hline \multirow[t]{3}{*}{ TS-6 } & 2001 & P. densiflora & 8 & 84 & 214 & 135 & 155 \\
\hline & & & 15 & 76 & 214 & 135 & 155 \\
\hline & & & 13 & $76 / 84$ & 214 & 135 & 155 \\
\hline \multirow[t]{4}{*}{ TS-7 } & 2001 & P. densiflora & 20 & 86 & 214 & 135 & 155 \\
\hline & & & 8 & 86 & 214 & 135 & $147 / 155$ \\
\hline & & & 8 & 86 & 338 & 135 & 155 \\
\hline & & & 3 & $78 / 86$ & $214 / 338$ & 135 & 155 \\
\hline TS-8 & 2001 & P. densiflora & 26 & 76 & 214 & 135 & 155 \\
\hline \multirow[t]{3}{*}{ TS-9 } & 2001 & P. densiflora & 8 & 72 & $214 / 320$ & 135 & 155 \\
\hline & & & 14 & 72 & 320 & 135 & 155 \\
\hline & & & 8 & 72 & 214 & 135 & 155 \\
\hline \multirow[t]{2}{*}{ TS-10 } & 2001 & P. densiflora & 21 & 84 & 214 & 135 & 155 \\
\hline & & & 5 & $76 / 84$ & 214 & 135 & 155 \\
\hline \multicolumn{8}{|l|}{ Chiba } \\
\hline $\mathrm{CH}-1$ & 2001 & P. densiflora & 48 & 84 & 214 & 135 & 155 \\
\hline $\mathrm{CH}-2$ & 2001 & P. densiflora & 30 & 108 & 214 & 131 & 155 \\
\hline \multirow[t]{4}{*}{$\mathrm{CH}-3$} & 2001 & P. densiflora & 2 & $84 / 108$ & $196 / 214$ & 135 & 155 \\
\hline & & & 3 & 108 & 214 & 135 & 155 \\
\hline & & & 12 & 84 & 196 & 135 & 155 \\
\hline & & & 10 & 84 & 214 & 135 & 155 \\
\hline $\mathrm{CH}-4$ & 2001 & P. densiflora & 28 & - & 212 & 135 & 155 \\
\hline CH-5 & 2001 & P. densiflora & 35 & 86 & 228 & 135 & 157 \\
\hline $\mathrm{CH}-6$ & 2001 & P. densiflora & 28 & 76 & 214 & 135 & 155 \\
\hline $\mathrm{CH}-7$ & 2001 & P. densiflora & 13 & 78 & $230 / 336$ & 137 & 155 \\
\hline & & & 9 & 78 & 230 & 137 & 155 \\
\hline & & & 4 & 78 & 336 & 137 & 155 \\
\hline CH-8 & 2001 & P. thunbergii & 40 & 78 & - & 135 & 155 \\
\hline CH-9 & 2001 & P. thunbergii & 15 & 78 & 214 & 135 & $147 / 155$ \\
\hline & & & 8 & 78 & 214 & 135 & 155 \\
\hline & & & 3 & 78 & 214 & 135 & 147 \\
\hline
\end{tabular}

${ }^{a}$ Four markers (Bx02, Bx03, Bx04, and Bx07) were used for genotypic polymorphism analyses of individual B. xylophilus.

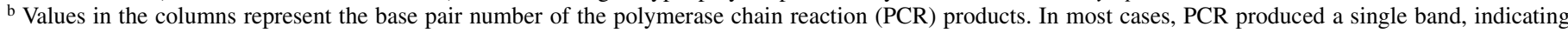
that the locus was homozygous, and only one value is listed. In a few cases, PCR produced two bands, indicating that the locus was heterozygous, and two values separated by a slash are listed. A dash indicates that no band was produced.

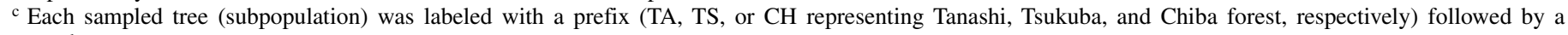
number. 
gosity of all markers (Table 3 ). The homozygous band sizes of $\mathrm{Bx} 02, \mathrm{Bx} 03, \mathrm{Bx} 04$, and $\mathrm{Bx} 07$ were 84, 320, 135, and $155 \mathrm{bp}$, respectively. Although the two exceptional individuals in subpopulation TA-12 had the same monomorphic allele composition at three loci as the other individuals, one showed a different homozygous allele pair of $322 \mathrm{bp}$ at locus $\mathrm{Bx} 03$, and the other showed a heterozygous allele pair of 84 and $76 \mathrm{bp}$ at $\mathrm{Bx} 02$. Two nematode subpopulations (TA-13 and TA-14) from the temple garden $\approx 0.7 \mathrm{~km}$ from the Experimental Station also showed the same genotype as subpopulations from the Experimental Station.

Genetic structure of nematode subpopulations in Tsukuba and Chiba. Ten and nine nematode subpopulations were extracted from dead pine trees in Tsukuba and Chiba, respectively. Genotypes of 11 to 48 individuals in each subpopulation were analyzed using the four markers. Five alleles for $\mathrm{Bx} 02$, three for $\mathrm{Bx} 03$, two for $\mathrm{Bx} 04$, and two for $\mathrm{Bx} 07$ were detected in B. xylophilus from Tsukuba (Table 3). Five alleles for $\mathrm{Bx} 02$, six for $\mathrm{Bx} 03$, three for $\mathrm{Bx} 04$, and three for $\mathrm{Bx} 07$ were detected in B. xylophilus from Chiba (Table 3).

Individuals from 10 subpopulations (TS-1, TS-3, TS-4, TS-8, $\mathrm{CH}-1, \mathrm{CH}-2, \mathrm{CH}-4, \mathrm{CH}-5, \mathrm{CH}-6$, and $\mathrm{CH}-8$ ) in Tsukuba and Chiba were monomorphic and homozygous at the four loci. Some

TABLE 4. Allele numbers and heterozygosities of the four microsatellite (SSR) markers in Bursaphelenchus xylophilus populations ${ }^{\mathrm{a}}$

\begin{tabular}{lccc}
\hline & & \multicolumn{2}{c}{ Heterozygosity $^{\mathrm{b}}$} \\
\cline { 3 - 4 } Locus & Allele number & Ho & He \\
\hline Bx02 & 6 & 0.028 & 0.578 \\
Bx03 & 9 & 0.052 & 0.638 \\
Bx04 & 4 & 0.000 & 0.173 \\
Bx07 & 3 & 0.026 & 0.143 \\
\hline
\end{tabular}

a Values were obtained from SSR genotypes of B. xylophilus from 33 subpopulations in three forests (Tanashi, Tsukuba, and Chiba). The genotypes were analyzed using four SSR markers (Bx02, Bx03, Bx04, and Bx07).

b The observed heterozygosity $(H \mathrm{o})$ at each SSR locus was obtained by adding $H \mathrm{o}$ values of all subpopulations and dividing by 33 , the number of subpopulations. The expected heterozygosity $(\mathrm{He})$ was calculated from allele frequencies at each SSR locus by Nei's equation. For calculating $\mathrm{He}$, each allele frequency was calculated by adding frequencies of the allele in all subpopulations and dividing by the number of subpopulations. At each locus, Ho significantly deviated from $\mathrm{He}$ (i.e., deviated from HardyWeinberg equilibrium) as determined by a $\chi^{2}$ test at $P<0.05$. individuals in the other nine subpopulations were heterozygous. Six (TS-5, TS-6, TS-9, TS-10, CH-7, and CH-9) were heterozygous for only one locus (Bx02, Bx03, or Bx07), two (TS-2 and $\mathrm{CH}-3)$ were heterozygous for two loci ( $\mathrm{Bx} 02$ and $\mathrm{Bx} 07$, or $\mathrm{Bx} 02$ and $\mathrm{Bx} 03$ ), and one (TS-7) was heterozygous for three loci (Bx02, Bx03, and Bx07) (Table 3).

In these two forests, 29 genotypes (16 from Tsukuba and 15 from Chiba) were distinguished, and the major genotype in each subpopulation differed from that in Tanashi (Table 3). Two individuals from a subpopulation in Tsukuba (TS-5) showed the same genotype as that in Tanashi. Several genotypes were shared by subpopulations in Tsukuba and Chiba. One genotype was found in four Tsukuba and two Chiba subpopulations (TS-3, TS5, TS-6, TS-10, CH-1, and CH-3). Another genotype occurred in two Tsukuba and one Chiba subpopulation (TS-6, TS-8, and CH$6)$.

The logs of the probabilities calculated by the STRUCTURE program were $-3,941(K=2),-2,992(K=3),-2,956(K=4)$, and $-2,482(K=5)$. Because it was the smallest value that captured the major structure in the data, a $K$ value of 3 was chosen (i.e., the nematode individuals were assigned to three origins [clusters], designated A, B, and C). Most of the nematodes from Tanashi were assigned to cluster A (Fig. 2). Although some individuals from two subpopulations in Tsukuba and one subpopulation in Chiba also were assigned to cluster $\mathrm{A}$, all nematodes from two subpopulations in Tsukuba and five in Chiba were assigned to cluster B with high probabilities. All nematodes from six subpopulations in Tsukuba and three in Chiba were assigned to cluster $\mathrm{C}$ with high probabilities. Interestingly, some subpopulations (TS-5, TS-9, and CH-3) contained nematode individuals in cluster $\mathrm{A}, \mathrm{C}$, and a mixed $\mathrm{A} / \mathrm{C}$ cluster. This may indicate that nematodes of cluster $\mathrm{A}$ and $\mathrm{C}$ were introduced to the same host tree, mated, and produced hybrid individuals.

Nematode genetic differences between subpopulations and between populations. A neighbor-joining dendrogram based on the genetic differences between subpopulations was created (Fig. 3). Nematode subpopulations in Tanashi belonged to a group that clearly was separated from those in Tsukuba and Chiba, but subpopulations in Tsukuba and Chiba were not separated from each other. In Chiba and Tsukuba, the genetic differences between subpopulations in the same forest were significantly larger than those between the two populations (Table 5). Genetic differences

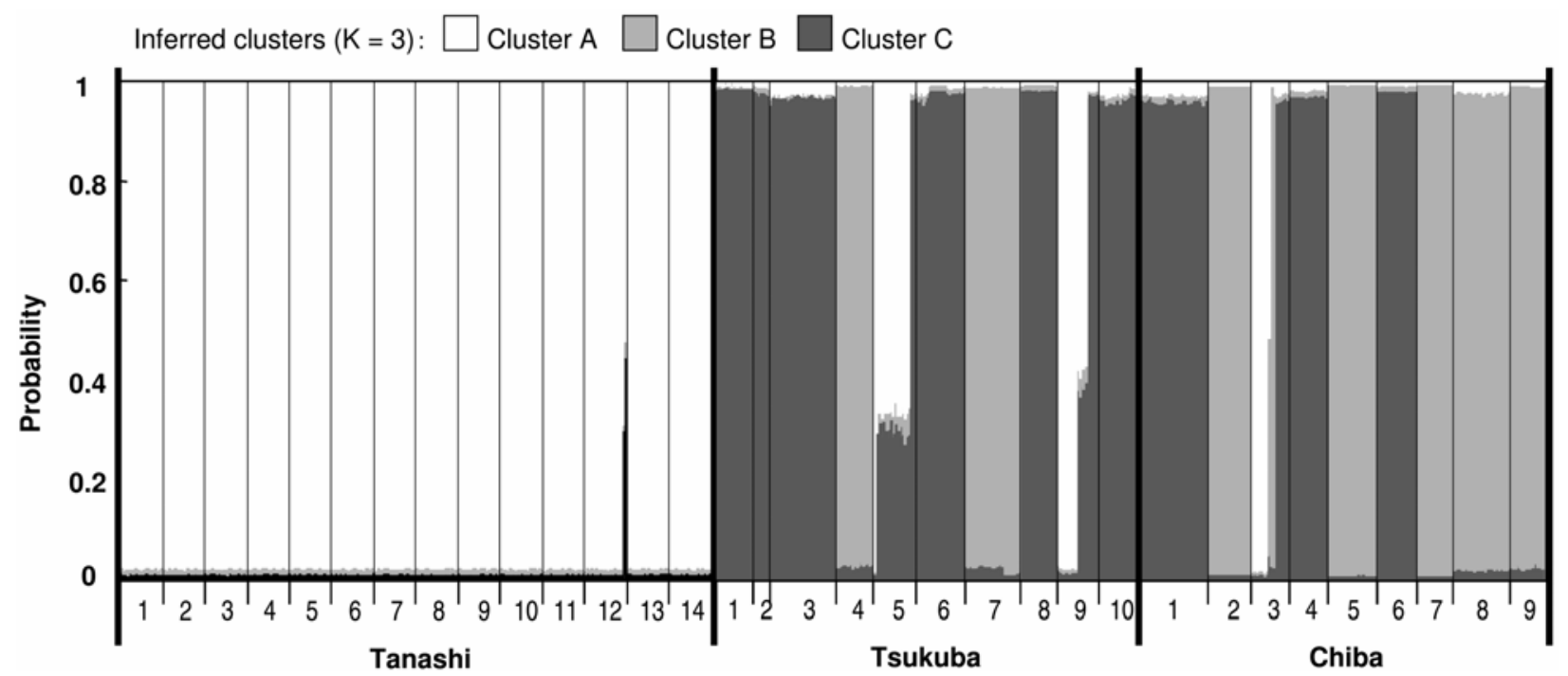

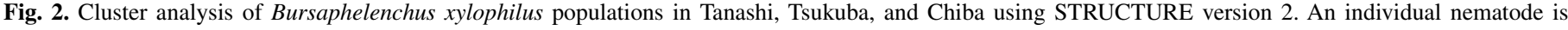

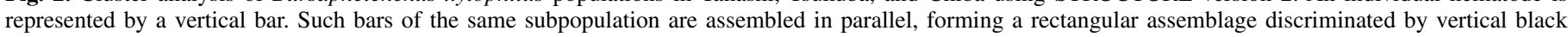

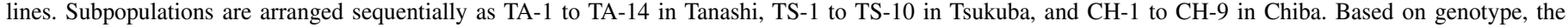

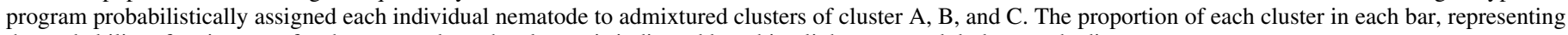
the probability of assignment for the nematode to the cluster, is indicated by white, light-gray, and dark-gray shading. 
between the Tanashi population and either Chiba or Tsukuba population were much larger than the genetic difference between the Chiba and Tsukuba populations. Genetic difference was not correlated with geographic distance among subpopulations in Tsukuba and Chiba.

\section{DISCUSSION}

Polymorphic and species-specific SSR markers of the pinewood nematode. Although the SSR markers that we developed for the pinewood nematode showed intraspecific polymorphism, the degree of SSR polymorphism seems rather small compared with that exhibited by SSR markers of other organisms $(11,14$, $21,23,28,29,31-33$ ). This low degree of polymorphism may partly result from $B$. xylophilus being an introduced species that has spread very rapidly through Japanese forests (27).

In this study, SSR markers were useful as intraspecific markers for identification of $B$. xylophilus genotypes. Because the nine nematode isolates used to test the makers were collected from a wide range throughout Japan, the genetic diversity among these isolates should well represent that of all B. xylophilus nematodes in Japan. In addition, PCR amplification of SSR markers for the pinewood nematode also was species specific to some extent. Several researchers previously have used PCR techniques other than SSR polymorphism analysis for specific and intraspecific identification of B. xylophilus $(1,3,4,7,9,10,18)$. The present SSR markers also could be used for $B$. xylophilus identification.

Genetic differences within and between nematode populations of the three forests. All nematode subpopulations extracted from dead pine trees in Tanashi had the same genotype, except for two individuals; this genotype was maintained for at least 2 years, suggesting that the same genotype has dominated the Tanashi region for years. Because Tanashi forest is fragmented and isolated from nearby forests by a large urban residential zone, this

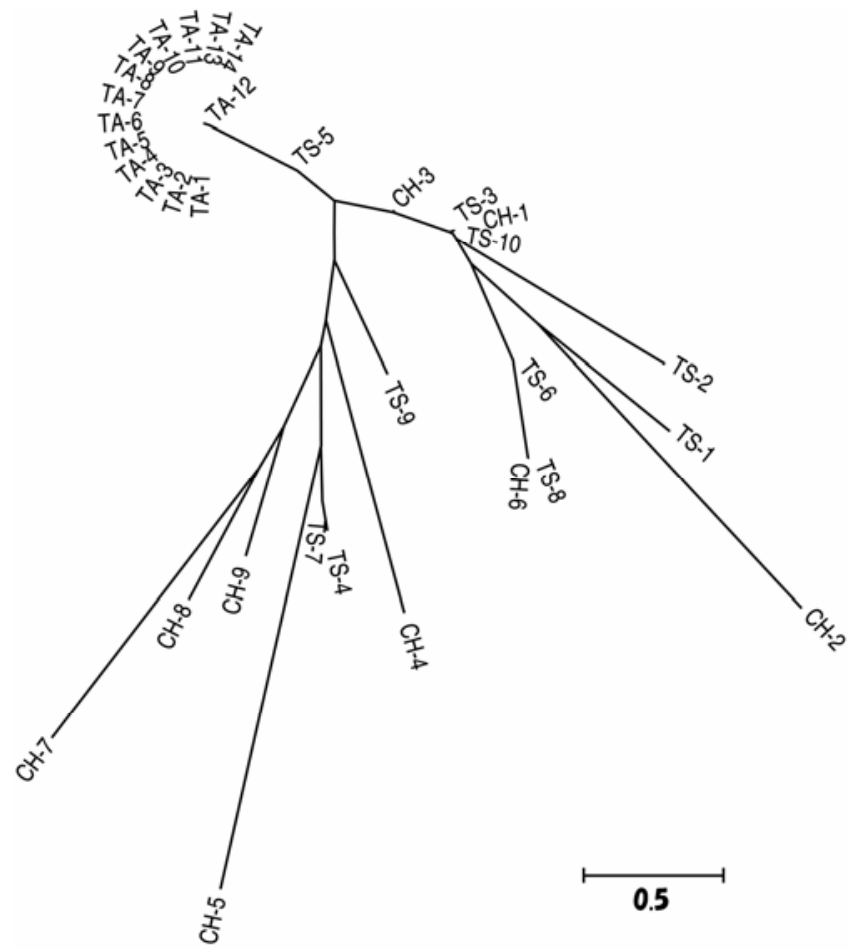

Fig. 3. Neighbor-joining dendrogram (using the Molecular Evolutionary Genetics Analysis software) based on the genetic differences between Bursaphelenchus xylophilus subpopulations in the three forests. Genetic differences were assessed using Nei's average number of differences, which were calculated using ARLEQUIN version 2. Every subpopulation was labeled with a prefix (TA, TS, or $\mathrm{CH}$ representing Tanashi, Tsukuba, and Chiba forests, respectively) followed by a number. genotypic simplicity may be the result of a founder effect and genetic drift. Pine wilt disease was first recognized in Tanashi in 1988; thus, the founder effect and genetic drift over the 13 intervening years may have resulted in genotypic simplification.

Compared with the Tanashi population, the Tsukuba and Chiba populations had substantially higher genotypic diversity. The Chiba and Tsukuba forests are larger and are less fragmented by residential regions than the Tanashi forest. Thus, the higher allele diversity may be attributable partly to larger nematode and sawyer beetle population sizes that reduced the founder effect and genetic drift.

The dendrogram based on genetic differences clearly distinguished Tanashi subpopulations from those in Tsukuba and Chiba, and the major genotype in Tanashi rarely was found in the other two forests. The genetic isolation of the Tanashi population from the other two populations may result from its geographic isolation (i.e., from its being surrounded by a large residential area). Because the spread of $B$. xylophilus relies on the migration of its vector, the pine sawyer beetle, the genetic isolation of the Tanashi nematode population may result from limited migration of the sawyer over the metropolis.

In contrast, the Tsukuba and Chiba subpopulations were intermingled in the dendrogram, and several genotypes commonly were observed in both forests. Because both Chiba and Tsukuba forests are large and seldom fragmented by residential regions, relatively large sawyer populations may inhabit these areas and migrate within and between the populations. This may result in significant nematode gene flow between the populations.

The cluster analysis suggests that nematodes in the three forests were derived from three different origins. The Tsukuba and Chiba nematode populations that were admixed with two clusters seem to have come from two origins (i.e., populations that differed from the Tanashi origin). This inference is consistent with the dendrogram (Fig. 3) showing that the Tanashi population is isolated from the Tsukuba and Chiba populations, and that the Tsukuba and Chiba populations are genetically indistinguishable.

Genetic difference between nematode subpopulations from individual trees. The genetic difference was much less within subpopulations than between subpopulations. Genotypic frequency distribution in each $B$. xylophilus subpopulation in a tree is highly affected by the genotypes of the founder nematodes introduced by the sawyer beetles and the mode of nematode proliferation within a tree. Nematodes are introduced into the tree via the wounds formed by sawyer maturation feeding (15). There is no report showing that the sawyer beetle keeps a territory for maturation feeding by driving other individuals from the tree. If a sawyer does not maintain such a territory after maturation feeding and if other sawyers can freely access the pine tree, the genotypic frequency distribution of nematodes would tend to be similar among trees. Thus, the mode of nematode introduction to the tree could not be the main cause of the relative genetic uniformity of individuals within the same tree.

TABLE 5. Genetic difference indices between and within Bursaphelenchus xylophilus populations ${ }^{\mathrm{a}}$

\begin{tabular}{lccc}
\hline & Tanashi & Tsukuba & Chiba \\
\hline Tanashi & $0.006^{\mathrm{b}}$ & $\ldots$ & $\ldots$ \\
Tsukuba & $0.955^{\mathrm{c}}$ & $1.259^{\mathrm{b}}$ & $\ldots$ \\
Chiba & $1.100^{\mathrm{c}}$ & $0.257^{\mathrm{c}}$ & $2.141^{\mathrm{b}}$ \\
\hline
\end{tabular}

${ }^{a}$ Genetic differences between and within three populations (Tanashi, Tsukuba, and Chiba) were assessed from microsatellite genotypes of individual nematodes using the ARLEQUIN program.

${ }^{b}$ Each diagonal element is the mean number of pairwise differences within the population, an index of genetic difference within the population.

${ }^{c}$ Each element below the diagonal is Nei's average number of differences between populations, an index of genetic difference between populations. For the calculation, the allele frequency in each forest was calculated as the average of frequencies of a given allele in all subpopulations in the forest. 
Experiments have shown that most B. xylophilus inoculated onto a pine tree are unable to invade the host pine tissues and those that do invade persist at an extremely low number until the beginning of symptom development $(16,17)$. Proliferation from such a small number of nematodes may produce a founder effect and early genetic drift, and result in a much simpler genotypic frequency distribution than that of the originally introduced nematodes.

Another remarkable characteristic of the B. xylophilus genotype was the excess of homozygous allele pairs. The Wahlund effect and inbreeding can explain an excess of homozygous allele pairs $(5,24,30)$. The Wahlund effect predicts that $H$ o is smaller than $H \mathrm{e}$ in a population under Hardy-Weinberg equilibrium in the presence of subpopulations with different allele frequencies. The present analyses of genetic differences and clusters between subpopulations indicated the independency between subpopulations. Therefore, the deviation between $\mathrm{Ho}$ and $\mathrm{He}$ could be mostly explained by the Wahlund effect. Because only a small number of nematodes may be present before the explosive multiplication stage, inbreeding also might contribute to the deviation.

\section{ACKNOWLEDGMENTS}

We thank T. Kiyohara, formerly of the Forestry and Forest Products Research Institute, Japan, for providing pinewood nematode isolates; and S. Ugawa, Graduate School of Frontier Sciences, the University of Tokyo, and T. Yonemichi, University Forest in Chiba, the University of Tokyo, for their support in collecting wood samples. This work was partly supported by a Grant-in-Aid for Scientific Research (16101008) from the Ministry of Education, Science, Sports, and Culture of Japan, and a grant (03DZ19317) from Science and Technology Commission of Shanghai Municipality, China.

\section{LITERATURE CITED}

1. Aikawa, T., Kikuchi, T., and Kosaka, H. 2003. Demonstration of interbreeding between virulent and avirulent populations of Bursaphelenchus xylophilus (Nematoda: Aphelenchoididae) by PCR-RFLP method. Appl. Entomol. Zool. 38:565-569.

2. Bergdahl, D. R., and Halik, S. 2001. Biology and ecology of pine wilt disease. Pages 229-238 in: Shade Tree Wilt Diseases. C. L. Ash, ed. The American Phytopathological Society, St. Paul, MN.

3. Cao, A.-X., Liu, X.-Z., Zhu, S.-F., and Lu, B.-S. 2005. Detection of the pinewood nematode, Bursaphelenchus xylophilus, using a real-time polymerase chain reaction assay. Phytopathology 95:566-571.

4. Castagnone, C., Abad, P., and Castagnone-Sereno, P. 2005. Satellite DNA-based species-specific identification of single individuals of the pinewood nematode Bursaphelenchus xylophilus (Nematoda: Aphelenchoididae). Eur. J. Plant Pathol. 112:191-193.

5. Castric, V., Bernatchez, L., Belkhir, K., and Bonhomme, F. 2002. Heterozygote deficiencies in small lacustrine populations of brook charr Salvelinus fontinalis Mitchill (Pisces, Salmonidae): A test of alternative hypotheses. Heredity 89:27-35.

6. Fukuda, K. 1997. Physiological process of the symptom development and resistance mechanism in pine wilt disease. J. For. Res. 2:171-181.

7. Iwahori, H., Kanzaki, N., and Futai, K. 2000. A simple, polymerase chain reaction-restriction fragment length polymorphism-aided diagnosis method for pine wilt disease. For. Pathol. 30:157-164.

8. Japanese Forestry Agency. 2005. On the annual loss of pines by pine wilt in 2004. Shinrinboeki (Forest Pests) 54:251-252. (in Japanese)

9. Kang, J.-S., Choi, K.-S., Shin, S.-C., Moon, I.-S., Lee, S.-G., and Lee, S.H. 2004. Development of an efficient PCR-based diagnosis protocol for the identification of the pinewood nematode, Bursaphelenchus xylophilus (Nematoda: Aphelenchoididae). Nematology 6:279-285.

10. Kanzaki, N., and Futai, K. 2002. A PCR primer set for determination of phylogenetic relationships of Bursaphelenchus species within the xylophilus group. Nematology 4:35-41.

11. Kawano, K. M. 2003. Isolation of polymorphic microsatellite markers in the great tit (Parus major minor). Mol. Ecol. Notes 3:314-315.

12. Kiyohara, T., and Suzuki, K. 1975. Seasonal population dynamics of Bursaphelenchus xylophilus in Pinus thunbergii trees. Mtg. Jpn. For. Soc. 86:296-298. (in Japanese)

13. Kumar, S., Tamura, K., Jakobsen, I. B., and Nei, M. 2001. MEGA2: Molecular evolutionary genetics analysis software. Bioinformatics 17:1244-1245.

14. Lian, C.-L., and Hogetsu, T. 2002. Development of microsatellite markers in black locust (Robinia pseudoacacia) using a dual-suppression-PCR technique. Mol. Ecol. Notes 2:211-213.

15. Linit, M. J. 1988. Nematode-vector relationships in the pine wilt disease system. J. Nematol. 20:227-235.

16. Mamiya, Y. 1983. Pathology of the pine wilt disease caused by Bursaphelenchus xylophilus. Annu. Rev. Phytopathol. 21:201-220.

17. Mamiya, Y. 1985. Initial pathological changes and disease development in pine trees induced by the pine wood nematode, Bursaphelenchus xylophilus. Ann. Phytopathol. Soc. Jpn. 51:546-555.

18. Matsunaga, K., and Togashi, K. 2004. A simple method for discriminating Bursaphelenchus xylophilus and B. mucronatus by species-specific polymerase chain reaction primer pairs. Nematology 6:273-277.

19. Nei, M. 1987. Molecular Evolutionary Genetics. Columbia University Press, New York.

20. Nickle, W. R., ed. 1991. Manual of Agricultural Nematology. Marcel Dekker, New York.

21. Nurtjahjaningsih, I. L. G., Saito, Y., Lian, C.-L., Tsuda, Y., and Ide, Y. 2005. Development and characteristics of microsatellite markers in Pinus merkusii. Mol. Ecol. Notes 5:552-553.

22. Pritchard, J. K., Stephens, M., and Donnelly, P. 2000. Inference of population structure using multilocus genotype data. Genetics 155:945959.

23. Saito, Y., Lian, C.-L., Hogetsu, T., and Ide, Y. 2005. Development and characterization of microsatellite markers in Abies firma and interspecific amplification in other Japanese Abies species. Mol. Ecol. Notes 5:234235.

24. Sánchez-Pérez, R., Martínez-Gómez, P., Dicenta, F., Egea, J., and Ruiz, D. 2006. Level and transmission of genetic heterozygosity in apricot (Prunus armeniaca L.) explored using simple sequence repeat markers. Genet. Resour. Crop Evol. 53:763-770.

25. Schneider, S., Roessli, D., and Excoffier, L. 2000. Arlequin ver. 2.000: A software for population genetics data analysis. Genetics and Biometry Laboratory, University of Geneva, Switzerland.

26. Siebert, P. D., Chenchik, A., Kellogg, D. E., Lukyanov, K. A., and Lukyanov, S. A.1995. An improved PCR method for walking in uncloned genomic DNA. Nucleic Acids Res. 23:1087-1088.

27. Suzuki, K. 2004. Pine wilt and the pine wood nematode. Page 773-777 in: Encyclopedia of Forest Sciences. J. Burley, J. Evans, and J. A. Youngquist, eds. Elsevier, Oxford.

28. Terui, H., Lian, C.-L., Saito, Y., and Ide, Y. 2006. Development of microsatellite markers in Acer capillipes. Mol. Ecol. Notes 6:77-79.

29. Wadud, M. A., Lian, C.-L., Nara, K., Ishida, T. A., and Hogetsu, T. 2006. Development of microsatellite markers from an ectomycorrhizal fungus, Laccaria amethystina, by a dual-suppression-PCR technique. Mol. Ecol. Notes 6:130-132.

30. Wahlund, S. 1928. Zusammensetzung von Populationen und Korrelationserscheinungen vom Standpunkt der Vererbungslehre ausbetrachtet. Hereditas 11:65-106.

31. Watanabe, A., Iwaizumi, M. G., Ubukata, M., Kondo, T., Lian, C.-L., and Hogetsu, T. 2006. Isolation of microsatellite markers from Pinus densiflora Sieb. et Zucc. using a dual PCR technique. Mol. Ecol. Notes 6:80-82.

32. Wu, B.-Y., Lian, C.-L., and Hogetsu, T. 2002. Development of microsatellite markers in white birch (Betula platyphylla var. japonica). Mol. Ecol. Notes 2:413-415.

33. Wu, B.-Y., Nara, K., and Hogetsu, T. 2005. Genetic structure of Cenococcum geophilum populations in primary successional volcanic deserts on Mount Fuji as revealed by microsatellite markers. New Phytol. 165:285-293. 